\title{
Synthesis of Thermo-Sensitive Poly( $N$-alkylacrylamide) Gel by Amidation of Poly(acrylic acid) Gel
}

\author{
Takashi IIZAWA, ${ }^{\dagger}$ Yoshinobu MATSUURA, Kazuya HAshidA, and Yukio OnOHARA \\ Department of Chemical Engineering, Faculty of Engineering, Hiroshima University, \\ Kagamiyama, Higashi-Hiroshima 739-8527, Japan
}

(Received June 11, 2003; Accepted August 4, 2003)

\begin{abstract}
KEY WORDS Thermo-Sensitive Polymer Gel / 1,8-Diazabicyclo-[5,4,0]-7-undecene / Amidation / Poly(acrylic acid) Gel / Poly $(N$-alkylacrylamide) Gel /
\end{abstract}

Poly( $N$-isopropylacrylamide) (PNIPA) gel is famous thermo-sensitive polymer gel which has lower critical solution temperature (LCST) in the vicinity of $32{ }^{\circ} \mathrm{C} .{ }^{1-3}$ Since it shows relatively quick and large volume change caused by a small temperature change around LCST and its monomer $N$-isopropylacrylamide (NIPA) is commercially available, many applications of PNIPA gel were proposed. ${ }^{4-7}$ However, the applications may be restricted because the operation temperatures depend on the LCST and cannot freely be configured. Therefore, polymer gels which show different LCST from and equal thermo-sensitivity to PNIPA gel are needed for practical use. Ito $^{8}$ reported LCST of numerous linear poly( $N$-alkylacrylamide)s and poly $(N, N$-dialkylacrylamide)s. The thermo-sensitive behavior of some poly ( $N$-alkylacrylamide) gels such as poly( $N$-ethylacrylamide) gel $^{9}$ and PNIPA gel was also observed. The LCST of poly( $N$-alkylacrylamide)s and the gels depends primarily on their chemical structure. However, the swelling behavior of gel was strongly affected by the network structure as well as the chemical structure. Since poly ( $N$-alkylacrylamide) gels with the same network structure were not prepared from the polymerization of the corresponding monomers, relative thermo-sensitivity of poly ( $N$-alkylacrylamide) gels could not been determined.

Recently, we have studied heterogeneous reaction of poly(acrylic acid)-1,8-diazabicyclo-[5,4,0]-7-undecene (DBU) salt (DAA) with some reagents such as alkyl halide and alkylamine. ${ }^{10-14}$ It is found that almost quantitative amidation of DAA with isopropylamine (IPA) occurred in the presence of triphenylphosphite (TPP) as an activating agent to give PNIPA gel. The reaction of DAA with alkylamines would be expected to proceed without any relation to structure of alkylamines

${ }^{\dagger}$ To whom correspondence should be addressed. and give poly( $N$-alkylacrylamide) gels with the same network structure.

In this communication, a series of thermo-sensitive poly( $N$-alkylacrylamide) gels containing $\mathrm{C}_{3}$ alkyl chains with the same network structure is prepared from the amidation of DAA with alkylamines such as $n$-propylamine (NPA), IPA, allylamine (AA), cyclopropylamine (CPA), and mixtures of NPA and IPA (Scheme 1). Also, we discuss LCST and relative thermo-sensitivity of the obtained polymer gels.

\section{EXPERIMENTAL}

Cylindrical DAA (length and diameter; about $5 \mathrm{~mm}$ ) was prepared via two-steps; the copolymerization of acrylic acid with $0.5 \mathrm{~mol} \%$ of $N, N^{\prime}$ methylenebisacrylamide in Teflon tubes (internal diameter; $6 \mathrm{~mm}$ ) and neutralization of the resulting gel with excess DBU in methanol according to the previous papers. ${ }^{12,13}$ The samples were sufficiently washed with methanol by a Soxhlet extractor, and dried in vacuo at $60^{\circ} \mathrm{C}$ until constant weight.

A typical amidation of DAA is as follows. Mixed solution $(50 \mathrm{~mL})$ of NPA $\left(2.0 \mathrm{~mol} \mathrm{~L}^{-1}\right)$ and TPP $\left(1.0 \mathrm{~mol} \mathrm{~L}^{-1}\right)$ in $N$-methyl-2-pyrrolidone (NMP) was charged into a $50 \mathrm{~mL}$ simple pressure vessel in a water bath at $80^{\circ} \mathrm{C}$. Some samples of cylindrical DAA were dipped in the solution. After the disappearance of the core, the reacted samples were washed away unreacted substances and by-products with methanol and acetonitrile by a Soxhlet extractor. They were dried in vacuo at $60^{\circ} \mathrm{C}$ until constant weight.

Equilibrium swelling ratio and swelling rate of gel were measured according the method reported. ${ }^{14}$ The equilibrium swelling ratio is defined as $\left(W_{\mathrm{s}}+W_{\mathrm{p}}\right) / W_{\mathrm{p}}$, where $W_{\mathrm{s}}$ and $W_{\mathrm{p}}$ are weights of absorbed water and 


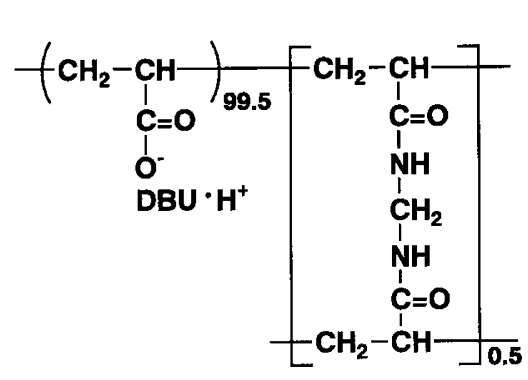

DAA

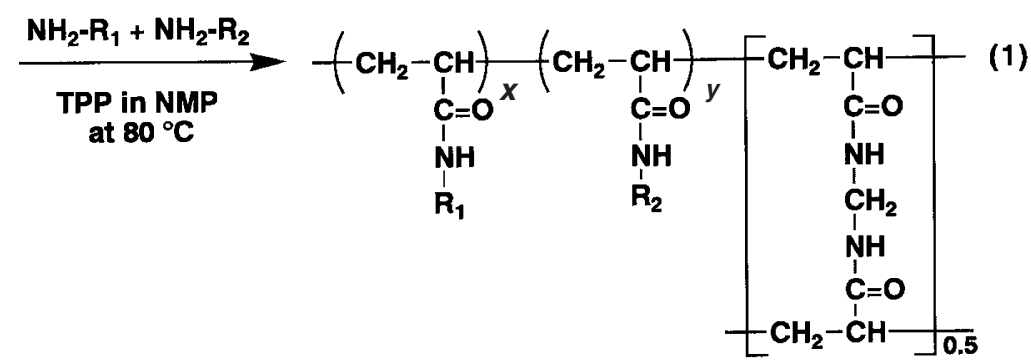

G-1 - G-9

$\mathrm{NH}_{2}-\mathrm{R}_{1}, \mathrm{NH}_{2}-\mathrm{R}_{2}: \mathrm{NH}_{2}-\mathrm{CH}_{2} \mathrm{CH}_{2} \mathrm{CH}_{3}$ (NPA), $\mathrm{NH}_{2}-\mathrm{CH}\left(\mathrm{CH}_{3}\right)_{2}$ (IPA), $\mathrm{NH}_{2}-\mathrm{CH}_{2} \mathrm{CH}=\mathrm{CH}_{2}$ (AA),

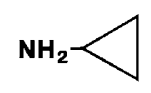

(CPA)

$\mathrm{P}\left(\mathrm{OC}_{6} \mathrm{H}_{5}\right)_{3}$

(TPP)<smiles>C1CCN2CCCN=C2C1</smiles>

(DBU)

Scheme 1. Reaction of DAA with Various Alkylamines using TPP.

Table I. Synthesis of poly( $N$-alkylacrylamide $)$ from reaction of DAA with alkylamine

\begin{tabular}{llccc}
\hline Gel & Alkylamine & $\begin{array}{c}\text { Content of IPA } \\
(\mathrm{mol} \%)\end{array}$ & Solvent & $\begin{array}{c}\text { Content of NIPA }^{\mathrm{b}} \\
(\mathrm{mol} \%)\end{array}$ \\
\hline G-1 & NPA & 0 & NMP & \\
G-2 & IPA & 100 & NMP & 99 \\
G-3 & AA & 0 & NMP & \\
G-4 & CPA & 0 & NMP & \\
G-5 & NPA + IPA & 10 & NMP & - \\
G-6 & NPA + IPA & 30 & NMP & 17 \\
G-7 & NPA + IPA & 50 & NMP & 33 \\
G-8 & NPA + IPA & 70 & NMP & 53 \\
G-9 & NPA + IPA & 90 & NMP & 83 \\
\hline
\end{tabular}

${ }^{a}$ Reaction of DAA was carried out with a mixture solution of TPP $\left(1.0 \mathrm{~mol} \mathrm{~L}^{-1}\right)$ and alkylamine $\left(2.0 \mathrm{~mol} \mathrm{~L}^{-1}\right)$ in NMP $(50 \mathrm{~mL})$ at $80^{\circ} \mathrm{C}$ for $1000 \mathrm{~min} .{ }^{\mathrm{b}} \mathrm{The}$ content of IPA in a mixture of IPA and NPA. ${ }^{c}$ The content of NIPA unit in the gel was estimated roughly by the IR spectrum.

dried polymer, respectively.

\section{RESULTS AND DISCUSSION}

Quantitative amidation of poly(acrylic acid) is very important to synthesize a thermo-sensitive $\operatorname{poly}(N$ alkylacrylamide) because unreacted acrylic acid units and by-products markedly affected its LCST., 13,15 In previous paper, ${ }^{14}$ we reported that the reaction of DAA with IPA using TPP gave a copolymer gel of NIPA with phenyl acrylate, and the content depended on the IPA concentration, although the apparent rate was not affected by the concentration. The amidation proceeded almost quantitatively when a high concentration of IPA solution was used. For example, the reaction of DAA with IPA $\left(1.0 \mathrm{~mol} \mathrm{~L}^{-1}\right)$ in NMP at $80^{\circ} \mathrm{C}$ gave a G$\mathbf{2}^{\prime}$ with $96.2 \mathrm{~mol} \%$ degree of amidation. ${ }^{14}$ According to the previous paper, ${ }^{14}$ the amidation of a cylindrical DAA (length the same as diameter; about $5 \mathrm{~mm}$ ) with various alkylamines $\left(2.0 \mathrm{~mol} \mathrm{~L}^{-1}\right)$ was carried out in NMP using TPP at $80^{\circ} \mathrm{C}$. When DAA was placed in NMP containing excess amounts of alkylamine and TPP, the swelling occurred and the gel was divided into a swelled shell and unswollen cylindrical core. The disappearing rate of the core is independent of kinds of alkylamines. After $1000 \mathrm{~min}$, the core disappeared. The complete reaction with various alkylamines such as NPA, IPA, AA, CPA, and mixture of NPA and IPA gave G-1, G-2, G-3, G-4, and G-5 G-9, respectively (Scheme 1 and Table I). The IR spectra of typical gels are shown in Figure 1. The spectrum of the obtained gels showed strong absorptions at 1650, 1545 (amide I and amide II), and $3300 \mathrm{~cm}^{-1}(\mathrm{~N}-\mathrm{H}$, stretching, and no absorption around $1755 \mathrm{~cm}^{-1}(\mathrm{C}=\mathrm{O}$ of phenyl ester $)$ was detected, although a trace absorption at $1755 \mathrm{~cm}^{-1}$ was observed in the spectra of G-2' with $96.2 \mathrm{~mol} \%$ degree of amidation. G-1, G-3, and G-4 showed characteristic absorptions at 1459,1384 , and $1153 \mathrm{~cm}^{-1}$ (n-propyl group), 1422, 1263, 996, and $923 \mathrm{~cm}^{-1}$ $\left(-\mathrm{CH}=\mathrm{CH}_{2}\right)$, and 1456,1362 , and $1026 \mathrm{~cm}^{-1}$ (cyclo- 


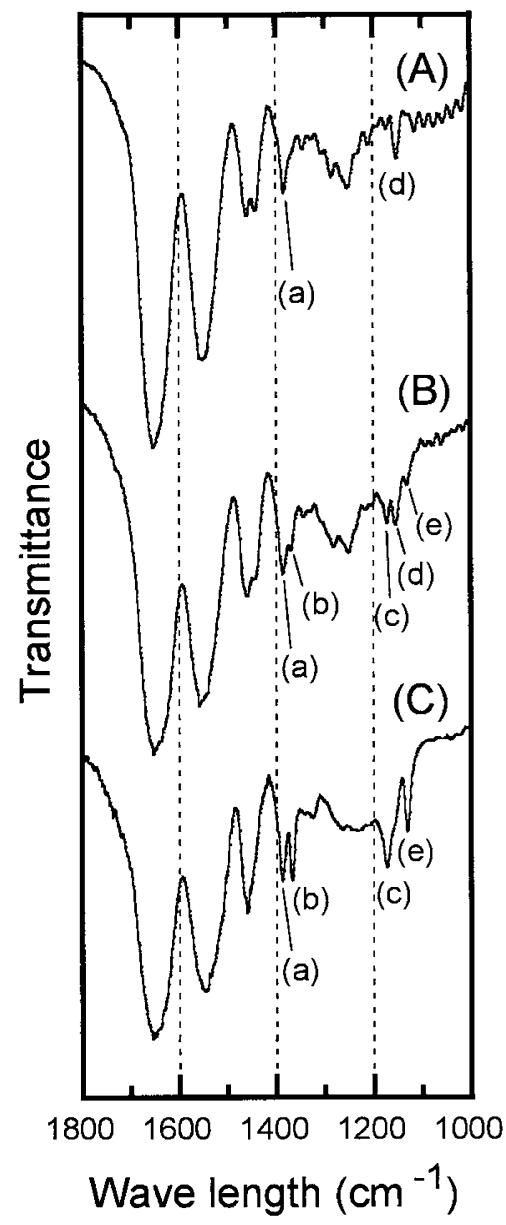

Figure 1. IR spectra ( $\mathrm{KBr}$ ) of (A) G-1, (B) G-7, and (C) G-2; (a) about $1385 \mathrm{~cm}^{-1}$, (b) $1364 \mathrm{~cm}^{-1}$, (c) $1173 \mathrm{~cm}^{-1}$, (d) $1153 \mathrm{~cm}^{-1}$, (e) $1131 \mathrm{~cm}^{-1}$.

propyl group), respectively. These spectra indicated that the almost quantitative amidation occurred to give the corresponding poly $(N$-alkylacrylamide) gels. In addition, poly $(N$-allylacrylamide) gel cannot be prepared from polymerization of $\mathrm{N}$-allylacrylamide because a radical copolymerization of acryl group and allyl group in $N$-allylacrylamide occurred competitively. G-7 prepared from the reaction of DAA with a mixture of NPA and IPA (1:1) showed absorptions at 1388, 1368, 1173, and $1131 \mathrm{~cm}^{-1}$ based on NIPA unit, and absorptions at 1384 and $1153 \mathrm{~cm}^{-1}$ based on $N$ - $n$-propylacrylamide (NNPA) unit. The IR spectra of G-5 G-9 consisted of those of G-1 and G-2. Therefore, these gels are copolymers of NIPA and NNPA. The content of NIPA units in the copolymer gels can be calculated from $A_{1131} / A_{\text {amide II }}$ or $A_{1153} / A_{\text {amide II }}$, where $A_{1131}, A_{1153}$, and $A_{\text {amide II }}$ are absorbances at $1131 \mathrm{~cm}^{-1}, 1153 \mathrm{~cm}^{-1}$, and $1545 \mathrm{~cm}^{-1}$, respectively, if the absorption coefficient of amide II at $1545 \mathrm{~cm}^{-1}$ based on NNPA units is the same as that based on NIPA units. The contents of NIPA units in G-6, G-7, and G-8 are about 17, 33, and $53 \mathrm{~mol} \%$, respectively, estimated roughly by this method, since the week characteristic absorptions were

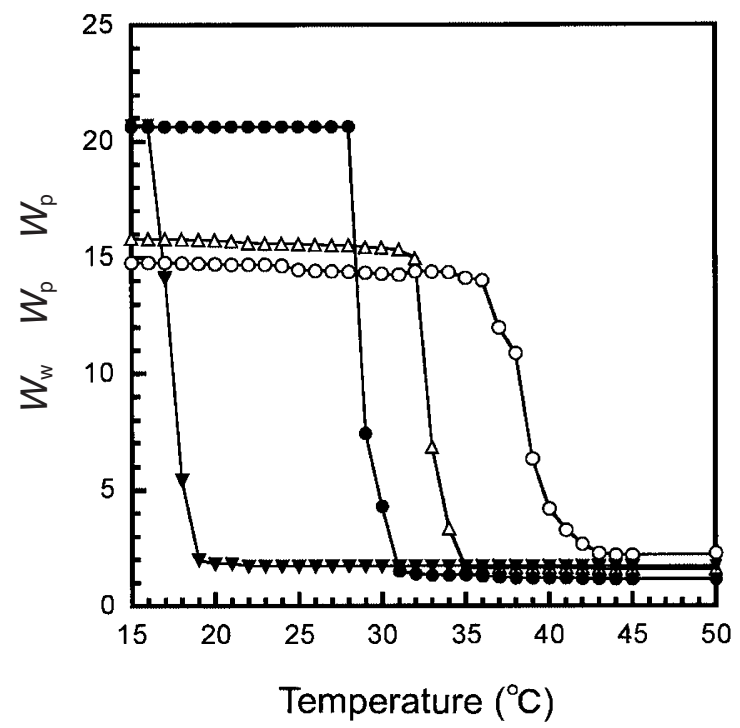

Figure 2. Equilibrium swelling ratio of $\operatorname{poly}(N$-alkylacrylamide) gels in water as a function of temperature; $(\mathbf{\nabla}) \mathbf{G}-\mathbf{- 1}$; G-2; $(\triangle)$ G-3; $(\bigcirc)$ G-4.

overlapped with the other absorptions. The content of NIPA units in the copolymer gels seems to be much lower than the bulky IPA content in the mixture of IPA and NPA because the heterogeneous reaction proceeded under diffusion control conditions. ${ }^{14}$

Equilibrium swelling ratio of G-1 $\sim$ G-4 was measured in water at a wide range of temperature (Figure 2). The swelling behavior of gel depended markedly on the network structure. It is difficult to prepare different gels with the same network structure from a polymerization method of monomer. On the other hand, the gels obtained from this synthetic method had the same network structure if the cleavage reaction or crosslinking reaction did not occur as a side reaction because the same lot of DAA was used. Therefore, the effect of alkyl group in $\operatorname{poly}(N-$ alkylacrylamide) gel on the equilibrium swelling ratio can be elucidated. Poly $(N$-alkylacrylamide) gel was hardly swollen in water when the water temperature was higher than the LCST. When the temperature became lower than the LCST, the gel was swelled and the equilibrium swelling ratio increased drastically, and finally the swelling ratio was independent of the temperature. The saturated equilibrium swelling ratio of G-1 (molecular formula of monomer unit: $\mathrm{C}_{6} \mathrm{H}_{11} \mathrm{NO}$ ) is the same as that of G-2 (molecular formula of monomer unit: $\mathrm{C}_{6} \mathrm{H}_{11} \mathrm{NO}$ ), and higher than those of $\mathbf{G - 3}$ and $\mathbf{G - 4}$ (molecular formula of monomer unit: $\mathrm{C}_{6} \mathrm{H}_{9} \mathrm{NO}$ ). G-1, G-2, G-3, and G-4 showed LCST at about 19, 31, 35, and $43{ }^{\circ} \mathrm{C}$. These LCSTs are slightly lower than those of the corresponding soluble polymers. ${ }^{8}$

It is well-known that gels having different LCST from PNIPA gel can be obtained from copolymeriza- 




Figure 3. Equilibrium swelling ratio of copolymer gels in water as a function of temperature; $(\mathbf{\nabla}) \mathbf{G}-\mathbf{1} ;(\bullet) \mathbf{G}-\mathbf{2} ;(\diamond) \mathbf{G}-\mathbf{5} ;(\triangle)$ G-6; (○) G-7; ( $\square)$ G-8; ( $\nabla)$ G-9.

tion of NIPA with other comonomers such as butyl methacrylate ${ }^{9}$ or $\mathrm{N}, \mathrm{N}$-dimethylacrylamide. ${ }^{15}$ A temperature change of LCST became larger with increasing the content of comonomer unit in the copolymer gel; however, it was so high that the gel showed low thermo-sensitivity. The equilibrium swelling ratio of copolymer gels G-5 G-9, compared with those of homopolymer gels G-1 and G-2, was shown in Figure 3. The copolymer gels showed LCST between those of G-1 $\left(19^{\circ} \mathrm{C}\right)$ and $\mathbf{G - 2}\left(31^{\circ} \mathrm{C}\right)$. The LCST increased with increasing the IPA content in the mixture of IPA and NPA in the synthesis of copolymer gels. Surprisingly, the saturated equilibrium swelling ratio was hardly changed by the content. When the dried gels were dipped in water at $15^{\circ} \mathrm{C}$, they swelled rapidly since they became hydrophilic under these conditions. The swelling rate was also independent of kinds of the gels. These results suggest that the copolymerization of NIPA and NNPA caused the LCST to change without decreasing the thermo-sensitivity. We cannot conclude at the present whether this copolymer system is special case because G-1 is structural isomer of G-2. Further studies on the amidation of DAA with other mixtures of alkylamines, the model reaction of soluble poly(acrylic acid) with the mixtures, and characteristics of the resulting polymers are now in progress.

Acknowledgments. The authors thank for a financial support from the Regional Research \& Development Consortium Project by Chugoku Bureau of Economy, Trade and Industry.

\section{REFERENCES}

1. M. Heskins and J. E. Guillet, J. Macromol. Sci., Chem., A2, 1441 (1968).

2. S. Hirotsu, Y. Hirokawa, and T. Tanaka, J. Chem. Phys., 87, 1392 (1987).

3. S. Fujishige, K. Kubota, and I. Ando, J. Phys. Chem., 93, 3311 (1989).

4. L. Dong and A. S. Hoffman, J. Controlled Release, 15, 141 (1990).

5. T. G. Park and A. S. Hoffman, J. Biomed. Mater. Res., 24, 21 (1990).

6. R. F. S. Frettas and E. L. Cussler, Chem. Eng. Sci., 42, 97 (1987).

7. Y. Seida and Y. Nakano, J. Chem. Eng. Jpn., 29, 767 (1996).

8. S. Ito, Kobunshi Ronbunshu, 46, 437 (1989).

9. T. Okano, Y. H. Bae, H. Jacobs, and S. W. Kim, J. Controlled Release, 11, 255 (1990).

10. T. Iizawa and F. Matsuda, Polym. J., 30, 155 (1998).

11. F. Matsuda, S. Miyamoto, and T. Iizawa, Polym. J., 31, 435 (1999).

12. F. Matsuda, N. Matsuno, and T. Iizawa, Kobunshi Ronbunshu, 55, 439 (1998).

13. T. Iizawa, N. Matsuno, M. Takauchi, and F. Matsuda, Polym. J., 31, 1277 (1999).

14. T. Iizawa, N. Matsuno, M. Takeuchi, and F. Matsuda, Polym. J., 34, 63 (2002).

15. Y. Seida, Y. Nakano, and H. Ichida, Kagaku Kogaku Ronbunshu, 20, 346 (1992). 